\title{
Histoplasma capsulatum causing sinusitis: a case report in French Guiana and review of the literature
}

\author{
C. Nabet ${ }^{1,3}$, C. Belzunce 2 , D. Blanchet ${ }^{1,3}$, P. Abboud $d^{2,3}$, F. Djossou ${ }^{2,3}$, B. Carme ${ }^{1,3}$, C. Aznar ${ }^{1,3}$ and M. Demar ${ }^{1,3^{*}}$ (D)
}

\begin{abstract}
Background: American histoplasmosis is a mycosis caused by Histoplasma capsulatum. A variety of clinical features of histoplasmosis have been commonly described ranging from asymptomatic infections to severe pulmonary infections. In immunocompromised individuals, progressive disseminated forms are frequent, leading to fatal outcome if untreated. However, H. capsulatum sinusitis is unusual with a few cases documented over the last three decades and may be underdiagnosed or confused with other fungal aetiologies, especially outside endemic regions.

Case presentation: In this study, we report an atypical case of Histoplasma capsulatum sinus fungus ball-like in a patient with Acquired Immunodeficiency Syndrome due to Human Immunodeficiency Virus complicated by a disseminated histoplasmosis with a death ending. Diagnosis relied on CT-Scan imaging and on both direct examination of $\mathrm{H}$. capsulatum yeast forms (Gomory methenamine Grocott) in the sinus specimen (aspirate, biopsy) and on positivity of the culture further confirmed by qPCR.

Conclusions: Since last few decades, among the eight reviewed patients, H. capsulatum sinusitis occurred mostly in HIV-immunocompromised patients and for three cases as a sinusitis with disseminated histoplasmosis. Even if this is a rare clinical presentation, its diagnosis is crucial as it could represent an early expression of an Histoplasma capsulatum exposure that can evolve into a disseminated fatal infection when immunity decreases.
\end{abstract}

Keywords: Histoplasma capsulatum, AIDS, Sinusitis, Sinus fungus ball, Review

\section{Background}

Sinusitis, also called rhinosinusitis and referred as symptomatic inflammation of the nasal and paranasal sinus mucosa, is a very common affection in general population. The most frequently reported pathogens are bacteria and viruses $[1,2]$. The recent well-defined entity named fungal rhinosinusitis whilst less prevalent is emerging, especially in tropical areas [3, 4]. Diverse clinical presentations and histopathology features of fungal rhinosinusitis are described based on the mode of fungal invasion of the tissues, probably depending on the variety of host interactions [5-8]. The non-invasive form

\footnotetext{
* Correspondence: magalie.demar@ch-cayenne.fr

'AcademicLaboratory of Parasitology and Mycology, Andrée Rosemon Hospital, 97306 Cayenne, French Guiana

${ }^{3}$ Faculty of Medicine, University of French Guiana, EA 3593 EPaT, Amazonian Ecosystems and Tropical Disease, Cayenne, French Guiana

Full list of author information is available at the end of the article
}

consists on fungall ball and allergic fungal rhinosinusitis whereas the invasive form includes acute, chronic, and chronic granulomatous rhinosinusitis. Fungal rhinosinusitis is commonly due to Aspergillus spp but other fungus species such as yeasts (Cryptococcus spp., Candida spp.), molds (Dematiaceous species, Rhizopus spp., Fusarium spp and Scedosporium spp.) have been reported mainly in HIV-AIDS patients [5, 6, 9, 10]. Few reports involving Histoplasma capsulatum (HC), an environmental and dimorphic fungus responsible for granulomatous disease are signed in the literature. This opportunist pathogen is isolated from endemic areas such as North, Central and South America, Africa, Asia and Australia with two pathogenic varieties in human, the worldwide $H$. capsulatum var. capsulatum and $H$. capsulatum var. duboisii isolated only in Africa [11].

The severity of the disease mainly depends on the inoculum size and the immunosuppression level allowing

(c) The Author(s). 2018 Open Access This article is distributed under the terms of the Creative Commons Attribution 4.0 International License (http://creativecommons.org/licenses/by/4.0/), which permits unrestricted use, distribution, and 
dissemination of the infection from lungs to lymph nodes or visceral involvement through the bloodstream [11-15]. This can lead to progressive disseminated histoplasmosis that can be fatal when therapy is delayed and when misdiagnosed [11, 14]. Though, rapid and accurate biological diagnostic of histoplasmosis is challenging as the absence of specific symptoms can lead to possible confusion with other opportunistic microbial agents such as tuberculosis [16]. This is even more obvious in case of atypical localizations. In French Guiana, histoplasmosis is known as a major opportunistic infection and one of the main causes of AIDS-related death, along with tuberculosis [14, 16-18]. First case of Histoplasmosis sinusitis was reported in 1993 from California, USA [19]. Globally, since the last three decades only 8 cases have been documented. Herein, such a case is reported in an HIV related-immunosuppressed adult and we then presented a comprehensive review of literature and analysed the previously reported cases.

\section{Case presentation}

\section{Clinical presentation}

A 50-year-old woman native of Haiti presented to the Cayenne hospital in French Guiana with complaints of persistent fever, mild cough, asthenia and anorexia. She reported medical consultation three months before for febrile nasal obstruction and cough treated by amoxicillin-clavulanic acid without ameliorations. She lived in French Guiana for 20 years.

Diagnosis of HIV infection was further achieved, showing a high viral replication level (HIV viral load $=7,300,000$ copies $/ \mathrm{mL}$ ) along with a deeply immunocompromised status (CD4 count $=22$ cells $/ \mathrm{mm} 3$ ). A full-body computer tomography scanner (CT scan) showed a complete opacification of right maxillary sinus filled with flocculent calcifications (Fig. 1). This typical aspect along with an absence of osteolysis or osteocondensation was evocative of an Aspergillus spp. fungus ball. Additionally, CT scan showed bilateral inferior alveolar opacities (also visible during chest radiography), hepatosplenomegaly and disseminated lymph nodes up to $2 \mathrm{cms}$.

\section{Clinical and laboratory findings}

Diagnosis protocol included for microbiological examinations: sputum, bronchoalveolar lavage (BAL), maxillary sinus puncture, bone marrow, osteomedullar, node and hepatitis biopsies. Potassium hydroxide $(\mathrm{KOH})$ direct examination and May-Grünwald Giemsa (MGG) stained smears were negative except for the sputum and the BAL. They showed an association of yeast and a gram-negative bacterium further identified in culture as Candida albicans, Klebsiella pneumonia and Pseudomonas fluorescens. Histopathology examination including Gomory Methenamine-Silver Grocott (GG) and Periodic Acid Schiff (PAS) staining

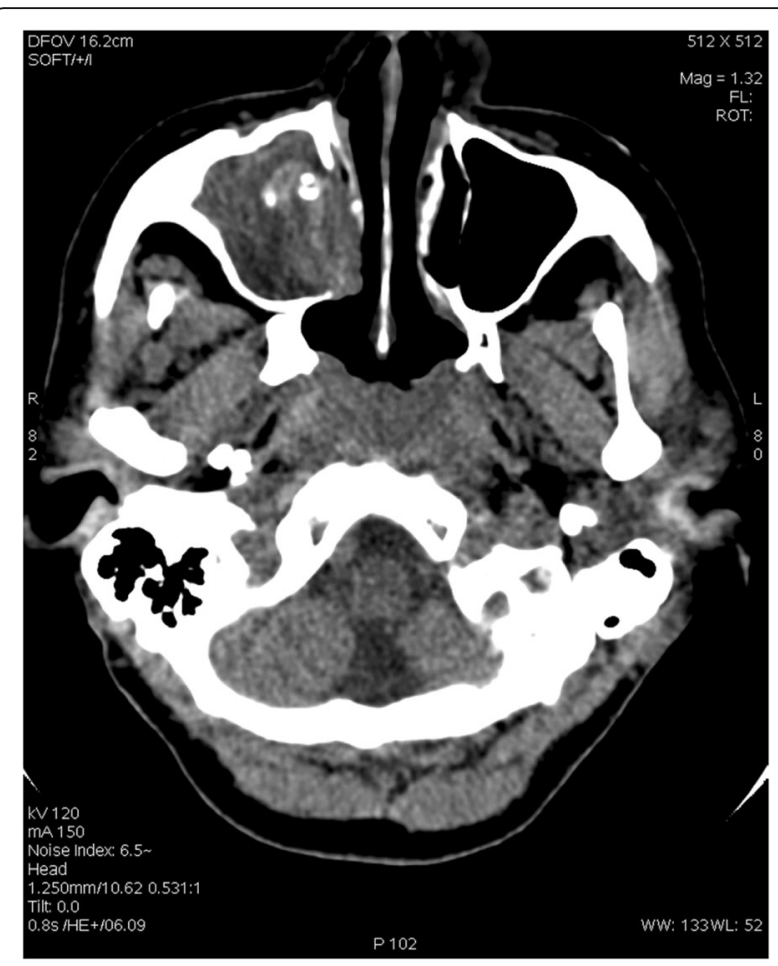

Fig. 1 Representative Computerized-Tomography Scan (CT-Scan) of the sinuses showing complete opacification of right maxillary sinus with flocculent calcifications and an absence of bone erosion

have been practiced on the liver, the osteomedullar biopsy and the BAL fluid and were negative. Serologic analysis revealed no detection of Aspergillus spp. galactomannan antigen nor specific antibodies for Histoplasma or Leishmania.

\section{Treatment and outcome of the patient}

Patient suddenly developed a respiratory distress syndrome along with a severe sepsis-like infection, after a prolonged well-supported fever. Antifungal therapy with Voriconazole was introduced due to presumption of an Aspergillus spp. maxillary infection that may be associated with pulmonary aspergillosis. Antimicrobial therapy with Piperacillin-tazobactam was introduced to cover a potential bacterial pulmonary infection (Klebsiella spp. and Pseudomonas spp.). Finally, the patient rapidly died of a respiratory distress syndrome, three days after the beginning of the pharmacological treatment and before the $H$. capsulatum infection was diagnosed.

\section{Confirmed diagnosis}

Indeed, $H$. capsulatum was isolated from the sinusitis aspirate and from cervical adenopathy biopsy after 20 and 15 days of culture, respectively using Sabouraud/ chloramphenicol/gentamicin agar media at $30{ }^{\circ} \mathrm{C}$. The other fungal cultures (bone marrow, liver biopsy, blood, urine, BAL and expectorations) remained sterile after 
2 months of incubation. Retrospectively, after a slide discoloration, a GG staining was performed on sinus aspirate and allowed to identify ovoid 2-4 $\mu \mathrm{m}$ yeasts evocative of Histoplasma capsulatum var. capsulatum, previously not visible during MGG staining (Fig. 2). Additionally, a $H$. capsulatum real-time PCR analysis developed by the Academic Laboratory of Parasitology and Mycology, Cayenne Hospital, French Guiana [20] was retrospectively performed on expectorations, BAL fluid and node biopsy and tested positive, confirming the diagnosis.

Several underlying pathologies have been revealed during post-mortem analysis. First, lymphoma diagnostic was evocated following direct examination of bone marrow, osteomedullar and node biopsies and was confirmed as a B lymphocyte-depleted Hodgkin's disease by immunophenotyping (CD20+ CD3+ CD30+ ki67+). Then, disseminated Mycobacterium avium infection was diagnosed by PCR on positive culturing of BAL, bone marrow, liver biopsy and node biopsy.

\section{Literature review}

A search for published cases of $H$. capsulatum sinusitis was conducted in PubMed database using the keywords "Histoplasma capsulatum", "sinusitis", "rhinosinusitis", "histoplasmosis". Only cases in which imaging confirmed sinusitis and $H$. capsulatum was documented by histopathologic direct examination or culture were included in the review. Studies in any language were considered and reviewed. Extracted data included age and gender, patient origin, underlying disease, clinical signs, symptoms duration, site of infection, direct examination and

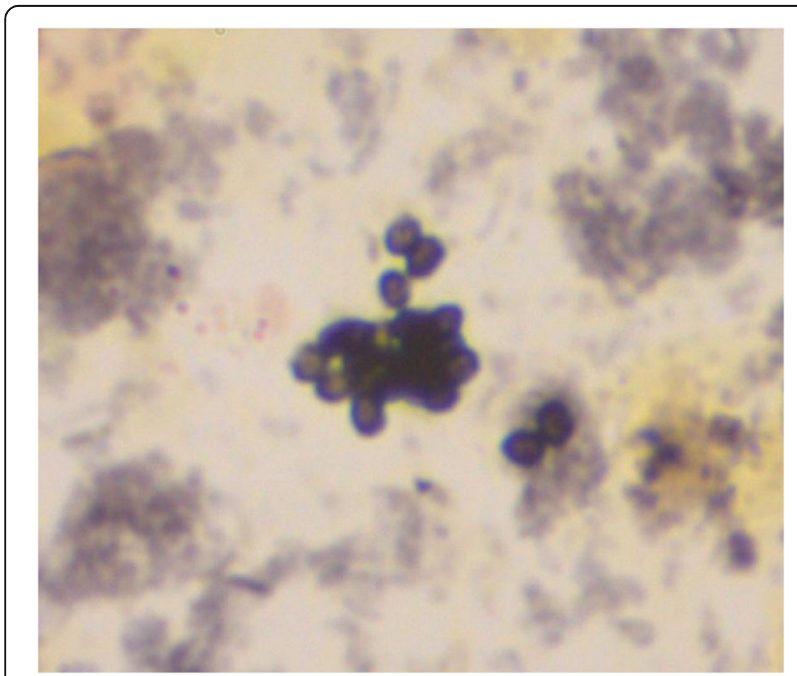

Fig. 2 Gomory Methenamine-Silver Grocott staining of the sinus aspirate showing ovoid 2-4 $\mu \mathrm{m}$ yeasts compatible with Histoplasma capsulatum var. capsulatum culture results, CT scan images of the sinus, treatment and outcome.

Eight articles reporting 8 cases of $H$. capsulatum sinusitis from 1993 to the present were included. These cases are summarized in Table 1 [19, 21-27].

\section{Discussions and conclusions}

In this study, fungal rhinosinusitis related to H. capsulatum is very uncommon and remains anecdotal as only eight cases have been published between 1993 and 2016. In the present case, it involves Histoplasma capsulatum var capsulatum and clinically presents as an atypical fungus ball-like in a deeply immunosuppressed patient with several co-morbidities.

\section{Risk factors for developing Histoplasma fungal rhinosinusitis and clinico-epidemiological aspects}

In our review, cases were reported from North America $(n=3)$, South America $(n=2)$, North Africa $(n=1)$, South Africa $(\mathrm{n}=1)$ and India $(\mathrm{n}=1)$. The mean age was 49 years, ranging from 35 to 90 years. Most cases of $H$. capsulatum sinusitis including the present case were described mostly in men (sex ratio H/F: 7/1) and immunocompromised host except for one of them (case 7). Indeed, six patients had HIV infection (cases 1, 2, 3, 4, 5, 8) and one a Chronic Lymphocytic Leukaemia (case 6). These features are in contrast with reported cases of typical sinus fungus ball. They usually occur in immunocompetent individuals and are more prevalent in women who had a prior history of dental surgery (iatrogenic oro-antral communication secondary to dental extraction, periodontal destruction or most often endodontic treatment with filling of the dental canal) [8-10].

According to the published sinus fungus ball findings, maxillary sinus is the most frequently involved sinus and multiple sinus locations are uncommon. In our review, other sinusal locations such as ethmoid and frontal were associated with the maxillary sinus for 5 cases $(3,4,5,6,8)$. Moreover, multiples sites of infection included the association of sinus/palate (cases 1, 2, 7), sinus/lung (case 5), sinus/skin (cases 3, 4, 8), sinus/cervical adenopathy/lung (present case). Only one case reported an unique sinusal location (case 6). Symptoms duration varied from 2 to 9 months (mean of 5 months) and main symptoms were fever $(n=5)$, headache $(n=4)$, nasal congestion $(n=4)$, rhinorrhoea $(n=3)$, palatal ulceration $(n=3)$, oral bleeding $(n=2)$, skin rash $(\mathrm{n}=3)$ and pneumopathy $(n=1)$.

\section{Pathogenesis of Histoplasma fungal rhinosinusitis}

Fungal sinusitis is divided into invasive and non-invasive forms $[8,10]$. Fungus ball of the paranasal sinuses is defined as the non-invasive accumulation of dense fungal concrements in sinusal cavities, most often the maxillary sinus. Clinicopathological criteria for diagnosis of fungus 


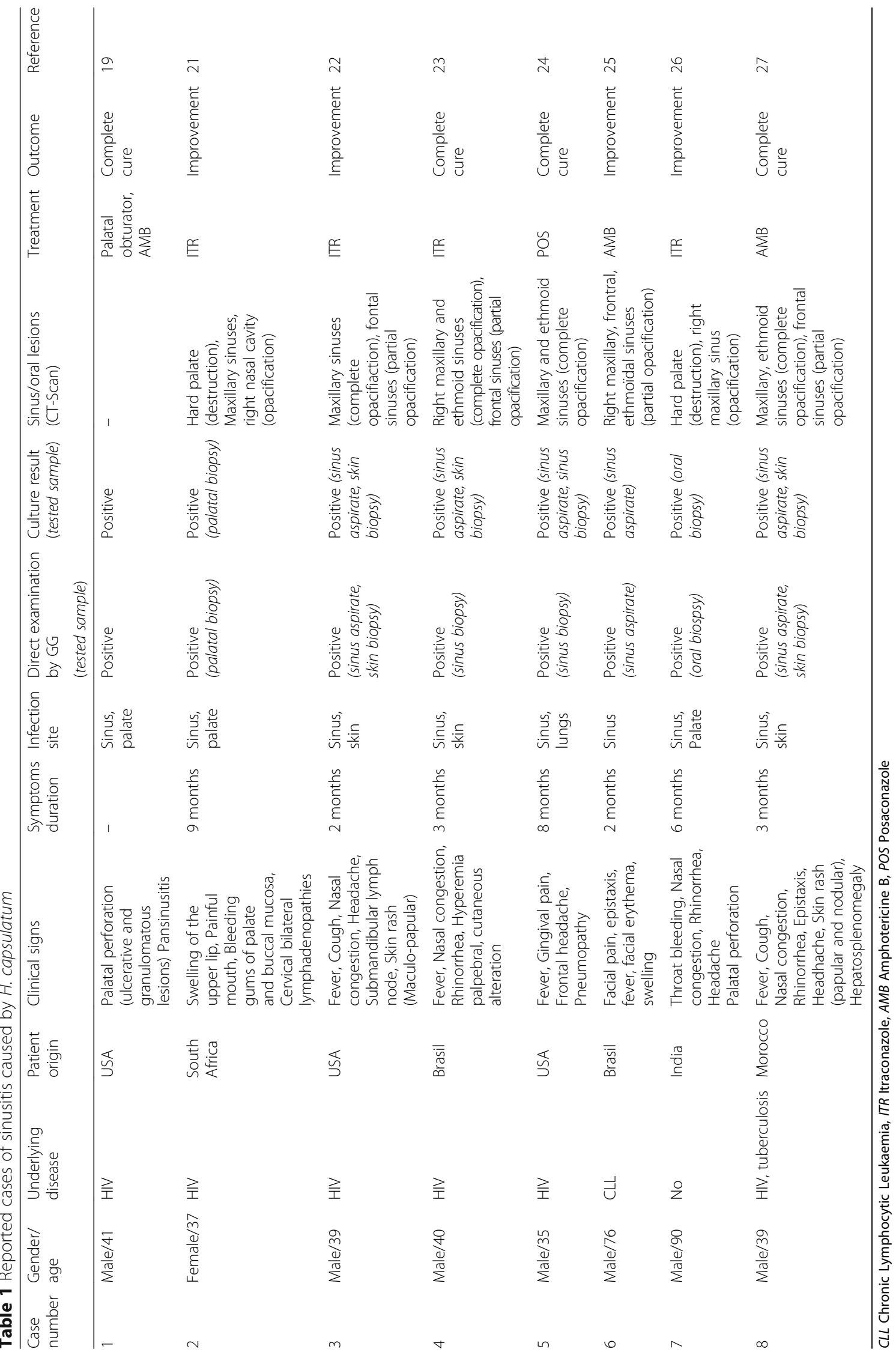


ball include, according to deShazo's et al. [28]: 1) Radiological evidence of sinus opacification with or without associated flocculent calcifications, 2) Mucopurulent, cheesy or clay-like material within a sinus, 3) a matted, dense conglomeration of hyphae separate from but adjacent to sinus respiratory mucosa 4) A chronic inflammatory response in the mucosa adjacent to fungal elements and 5) no histological evidence of fungal invasion of mucosa on GG.

Unlike invasive rhinosinusitis, sinus fungus ball never causes progression or invasion $[8,10]$. However, to explain the multiple sites of infection in 7 of the 8 reported cases $(1,5,7,8)$ and the present case, we could mention two different mechanisms. First, for non-contiguous sites, the dissemination may have resulted of a primary exposure to $H$. capsulatum by direct inhalation of spores. Probably, an acute pulmonary disease could have happened at exposure time and may have been initially recovered by immunity (granulomatous lesion). This was supposed for cases $3-5,8$ and present case that displayed a several months history of nasal obstruction, rhinorrhoea, headache and cough. A secondary progressive disseminated fungal infection was highly suspected for cases 3-5, 8 and present case, as patients presented fever, chest radiograph abnormalities $(3,5,8$, present case, not reported in Table 1), hepatosplenomegaly (8, present case), skin lesions $(3,4,8)$ and disseminated lymph nodes (8, present case). It was confirmed for cases $3,4,8$ and present case as $H$. capsulatum was found in culture from both sinus specimen and a specimen from a distant site (skin biopsy, cervical lymph node). In present case, $H$. capsulatum was retrospectively revealed on respiratory specimens with real-time PCR analysis; This pulmonary localisation may have been the potential source of fungus dissemination to cervical lymph node through the hematogenous route. The other mechanism for the development of the sinusitis may be a local extension from a contiguous source $[7,10]$. Indeed, for cases 1,2 and 7 , a prior palatal fungal ulcer may have eroded the hard palate and lead to secondary sinus infection by direct extension. When reviewing $H$. capsulatum sinusitis cases, no cases reporting with an odontogenic pathway mechanism have been described whereas it is a principal risk factor described for typical sinus fungus ball $[8,10]$.

\section{Challenge in the diagnosis and utility of new diagnostic tools}

Because histoplasmosis can have an extended incubation period and may goes undiagnosed for a long time before the worsening of symptoms, its diagnosis remains a real challenge. Moreover, the clinical presentation is misleading because usually non-specific and sometimes uncommon as reported in our review.
The present case emphasises the difficulties in diagnosing $H$. capsulatum from the direct examination. It highlights the better performance of GG staining for some samples, as we missed the diagnosis by using the MGG staining. In the review, direct microscopic examination of sinus aspirate (cases $3,6,8$ ) staining by GG easily revealed Histoplasma capsulatum yeasts. Additionally, $H$. capsulatum sinusitis may be confused with an Aspergillus spp. fungus ball. Indeed, our patient's radiological findings were strongly suggestive for an Aspergillus spp. sinus fungus ball showing complete opacification of maxillary sinus associated with flocculent calcifications. Another Aspergillus spp. fungus ball confusing element was the mucopurulent, clay-like aspect of the material within the sinus. Thus, in case of suggestive aspect of Aspergillus spp. fungus ball, it seems relevant to search for atypical fungal pathogens such as $H$. capsulatum, especially in immunocompromised host and in endemic areas.

We could not detail the fungus ball clinical form of the sinusitis because of insufficient histopathological criteria, especially for cases $3-6,8$ and the present one. However, the association of the imaging findings, the positivity of both fungal culture and direct examination of sinus aspirate and/or sinus biopsy along with sinusitis symptoms was consistent with a sinus fungus ball for our case. Similarly, for cases 3-5,8 with multiple distant infection site, even if we could not conclude about the invasive character of the sinusitis, we suspected a secondary dissemination from a pulmonary infection site rather than from a sinus infection, based on clinical symptoms chronology.

The culture confirmed the diagnosis for all the cases. We highlight that at least one culture of sinus specimen (sinus biopsy, sinus aspirate) tested positive for H. capsulatum in most reported cases. This result contrasts with the low positivity rate of fungal culture (between 23 and $50 \%$ ) observed from typical fungus balls, probably due to a poor viability of fungal elements in the fungus ball $[8,10]$. This current gold standard diagnostic test presents a positivity rate of more than $75 \%$ cases in disseminated Histoplasma capsulatum infections [11]. However, the long delay for the fungi culture could easily allow the routine use of the qPCR test for rapid diagnosis. This has not been possible in this present case as the home-made qPCR is performed for research only. However, its sensitivity has been assessed in small cohorts of patients and different outcomes have been observed when compared with other diagnostic methods [11]. As an alternative to PCR, cross reactivity with Aspergillus spp. galactomannan antigens in sera have also been reported to be useful for the diagnosis of disseminated histoplasmosis in AIDS patients in endemic areas where Histoplasma EIA is not available [29, 30]. Unfortunately, the test was negative for our case and not 
used in the previous case reports. Recently, detection of specific Histoplasma capsulatum galactomannan antigens in urine specimens during immunoenzyme assay (EIA) has been shown to be a promising test, highly sensitive and rapid [31]. This report highlights the need of new biological tools to improve and accelerate diagnosis of histoplasmosis.

\section{Treatment and outcome}

Antifungal treatment of sinusitis in the previously reported cases consisted in a long-term administration of Amphotericin B (cases 1, 6, 8), Itraconazole (cases 2, 3,4,7) and Posaconazole (case 5) leading to resolution of symptoms. No local surgery was required in contrast to typical sinusitis fungus balls that require endoscopic sinus surgery $[8,10]$. No death was reported. Recommended antifungal agents for histoplasmosis treatment are Amphotericin B (including liposomal and lipid complex formulations) and Itraconazole [12]. Posaconazole and Voriconazole also demonstrated in vitro activity against $H$. capsulatum and most studies have reported success with these azoles $[24,32]$. In present case, Voriconazole efficiency was difficult to evaluate as treatment was introduced too late with a curative dose for aspergillosis and the patient had too many concomitant life-threatening co-infections such as lymphoma and disseminated mycobacterium infection. This was the only $H$. capsulatum sinusitis case reporting patient death but association between $H$. capsulatum infection and death could not be demonstrated due to multiple co-morbidities.

In conclusion, histoplasmosis is a serious fatal risk for the AIDS-patients, requiring quick and reliable routine diagnostic methods. Moreover, there is a lack of specific recommendation for histoplasmosis prophylaxis in France, without any exception for French Guiana [33]. In opposition, in USA, a prophylaxis with Itraconazole $(200 \mathrm{mg}$ daily) is recommended in HIV infected patients with CD4 cell counts $<150$ cells $/ \mathrm{mm} 3$ in specific areas of endemicity [3]. A particular attention has to be given in AIDS-patients with sinusitis symptoms in $H$. capsulatum endemic areas such as in French Guiana. Despite the rarity of the clinical symptoms, sinusitis could represent an early expression of a $H$. capsulatum exposure. A sinus aspiration and/or a sinus biopsy should be performed rapidly for microbial analysis and essential as it can evolve into a disseminated infection when immunity decreases.

\section{Abbreviations}

AIDS: Acquired Immunodeficiency Syndrome; Bal: Bronchoalveolar lavage; CHAR: Centre Hospitalier Andrée Rosemon; CT- Scan: Computed tomography scan; ElA: Enzyme immuno-assay; GG: Gomory Methanamine-Silver Grocott staining; HIV: Human immunodeficiency virus; MGG: May Grunwald Giemsa; PCR: Polymerase Chain reaction

\section{Acknowledgements}

We acknowledge the staff of the Parasitology and Mycology Academic Laboratory as well as the staff of the Infectious diseases and Tropical medicine Unit, Andrée Rosemon Hospital, Cayenne, French Guiana.

\section{Funding}

No fundings.

\section{Availability of data and materials}

Data collection and sharing is not applicable to this article as no datasets were generated or analysed during the current study.

\section{Authors'contributions}

Conceptualization, Methodology CN, CB, MD The design of the article and its goals and aims. Development or design of methodology; creation of models. Data Curation CN, CB, MD Management activities to annotate data and maintain data for initial use and later reuse. Supervision MD Oversight and leadership responsibility for the design of the article. Visualization $C N, C B$, MD, FD, PA, CA, BC Preparation, creation and/or presentation of the published work, specifically visualization/data presentation. Writing - Original Draft Preparation CN, CB, MD Creation and/or presentation of the published work, specifically writing the initial draft. Writing - Review \& Editing CN, CB, MD, FD, PA, CA, BC Critical review, commentary or revision - including pre- or postpublication stages. All authors read and approved the final manuscript.

Ethics approval and consent to participate

Not applicable. Consent was obtained from the patient described in this report.

\section{Consent for publication}

Consent for publication was obtained from the individual patient described in this report. We obtain her sister's written consent. Written consents are available by request.

\section{Competing interests}

The authors declare that they have no competing interests.

\section{Publisher's Note}

Springer Nature remains neutral with regard to jurisdictional claims in published maps and institutional affiliations.

\section{Author details}

${ }^{1}$ AcademicLaboratory of Parasitology and Mycology, Andrée Rosemon Hospital, 97306 Cayenne, French Guiana. ${ }^{2}$ Unit of infectious diseases and Tropical medicine, Andrée Rosemon Hospital, Cayenne, French Guiana.

${ }^{3}$ Faculty of Medicine, University of French Guiana, EA 3593 EPaT, Amazonian Ecosystems and Tropical Disease, Cayenne, French Guiana.

Received: 6 June 2018 Accepted: 1 November 2018

Published online: 26 November 2018

\section{References}

1. Rosenfeld RM. CLINICAL PRACTICE. Acute sinusitis in adults. N Engl J Med 2016;375(10):962-970

2. Rosenfeld RM, Piccirillo JF, Chandrasekhar SS, Brook I, Ashok Kumar K, Kramper M, Orlandi RR, Palmer JN, Patel ZM, Peters A, Walsh SA, Corrigan MD. Clinical practice guideline (update): adult sinusitis. Otolaryngol Head Neck Surg. 2015; 152(2 Suppl):S1-S39. https://doi.org/10.1177/0194599815572097.

3. Singh AK, Gupta P, Verma N, Khare V, Ahamad A, Verma V, Agarwal SP. Fungal rhinosinusitis: microbiological and histopathological perspective. J Clin Diagn Res. 2017;11(7):DC10-2. https://doi.org/10.7860/JCDR/2017/25842. 10167. Epub 2017 Jul.

4. Krishnan KU, Agatha D, Selvi R. Fungal rhinosinusitis: a clinicomycological perspective. Indian J Med Microbiol. 2015;33(1):120-4. https://doi.org/10. 4103/0255-0857.148407.

5. Montone KT. Pathology of fungal rhinosinusitis: a review. Head Neck Pathol. 2016; 10(1):40-6. https://doi.org/10.1007/s12105-016-0690-0. Epub 2016 Feb 1. Review.

6. Montone KT, Livolsi VA, Feldman MD, Palmer J, Chiu AG, Lanza DC, Kennedy DW, Loevner LA, Nachamkin I. Fungal rhinosinusitis: a retrospective microbiologic and pathologic review of 400 patients at a single university medical center. Int J Otolaryngol. 2012;2012:684835. https://doi.org/10.1155/2012/684835. 
7. Jiang RS, Huang WC, Liang KL. Characteristics of sinus fungus ball: a unique form of rhinosinusitis. Clin Med Insights Ear Nose Throat. 2018;11:1179550618792254. https:/doi.org/10.1177/1179550618792254. eCollection 2018.

8. Grosjean P. Weber R. Fungus balls of the paranasal sinuses: a review. Eur Arch Otorhinolaryngol. 200;264(5):461-70.

9. Humphrey JM, Walsh TJ, Gulick RM. Invasive aspergillus sinusitis in human immunodeficiency virus infection: case report and review of the literature. Open Forum Infect Dis. 2016;3(3):ofw135.

10. Thompson GR 3rd, Patterson TF. Fungal disease of the nose and paranasal sinuses. J Allergy Clin Immunol. 2012;129(2):321-6. https://doi.org/10.1016/j. jaci.2011.11.039. Epub 2011 Dec 28. Review.

11. Wheat $L$, Azar MM, Bahr NC, Spec A, Relich RF, Hage C. Histoplasmosis. Infect Dis Clin N Am. 2016;30(1):207-27.

12. Wheat $L$, Freifeld AG, Kleiman MB, Baddley JW, McKinsey DS, Loyd JE, et al. Clinical practice guidelines for the management of patients with histoplasmosis: 2007 update by the Infectious Diseases Society of America. Clin Infect Dis. 2007;45(7):807-25.

13. Adenis AA, Aznar C, Couppie P. Histoplasmosis in HIV-infected patients: a review of new developments and remaining gaps. Curr Trop Med Rep 2014; 1:119-128, DOI: 10.1007/s40475-014-0017-8.

14. Nacher M, Adenis A, Blanchet D, Vantilcke V, Demar M, Basurko C, et al. Risk factors for disseminated histoplasmosis in a cohort of HIV-infected patients in French Guiana. PLoS Negl Trop Dis. 2014;8(1):e2638.

15. Assi MA, Sandid MS, Baddour LM, Roberts GD, Walker RC. Systemic histoplasmosis: a 15-year retrospective institutional review of 111 patients. Medicine (Baltimore). 2007;86(3):162-9.

16. Nacher M, Adenis A, Sambourg E, Huber F, Abboud P, Epelboin L, et al. Histoplasmosis or tuberculosis in HIV-infected patients in the amazon: what should be treated first? PLoS Negl Trop Dis. 2014;8(12):e3290.

17. Huber F, Nacher M, Aznar C, Pierre-Demar M, El Guedj M, Vaz T, et al. AIDSrelated Histoplasma capsulatum var. capsulatum infection: 25 years experience of French Guiana. AIDS. 2008;22(9):1047-53.

18. Couppie P, Aznar C, Carme B, Nacher M. American histoplasmosis in developing countries with a special focus on patients with HIV: diagnosis, treatment, and prognosis. Curr Opin Infect Dis. 2006;19(5):443-9.

19. Lucatorto F, Eversole LR. Deep mycoses and palatal perforation with granulomatous pansinusitis in acquired immunodeficiency syndrome: case reports. Quintessence Int. 1993;24(10):743-8.

20. Moquet O, Blanchet D, Simon S, Veron V, Michel M, Aznar C. Histoplasma capsulatum in Cayenne, French Guiana. Mycopathologia. 2012;174(4):331-4.

21. White J, Khammissa R, Wood NH, Meyerov R, Lemmer J, Feller L. Oral histoplasmosis as the initial indication of HIV infection: a case report. SADJ. 2007;62(10):452, 454-5.

22. Butt AA, Carreon J. Histoplasma capsulatum sinusitis. J Clin Microbiol. 1997; 35(10):2649-50

23. Araujo Filho BC, Campelo VE, Neves MC, Voegels RL, Butugan O. Histoplasmosis and rhinosinusitis: a rare association. Int Arch Otorhinolaryngol. 2006;10(2):141-5.

24. Restrepo A, Tobon A, Clark B, Graham DR, Corcoran G, Bradsher RW, et al. Salvage treatment of histoplasmosis with posaconazole. J Inf Secur. 2007; 54(4):319-27.

25. Alves MD, Pinheiro L, Manica D, Fogliatto LM, Fraga C, Goldani LZ. Histoplasma capsulatum sinusitis: case report and review. Mycopathologia. 2011;171(1):57-9.

26. Verma RK, Shankar A, Kumar Arora S, Sivaprakash MR, Panda NK. Histoplasma rhinosinusitis presenting as a nonhealing oral ulcer in an immunocompetent patient. J Otolaryngol Head Neck Surg. 2011:40(6):E47-50.

27. Elansari R, Abada R, Rouadi S, Roubal M, Mahtar M. Histoplasma capsulatum sinusitis: possible way of revelation to the disseminated form of histoplasmosis in HIV patients: Case report and literature review. Int I Surg Case Rep. 2016;24:97-100.

28. de Shazo RD, O'Brien M, Chapin K, Soto-Aguilar M, Swain R, Lyons M, et al. Criteria for the diagnosis of sinus mycetoma. J Allergy Clin Immunol. 1997; 99(4):475-85.

29. Iriart X, Blanchet D, Menard S, Lavergne RA, Chauvin P, Adenis A, et al. A complementary tool for management of disseminated Histoplasma capsulatum var. capsulatum infections in AIDS patients. Int I Med Microbiol. 2014;304(8):1062-5.

30. Riviere S, Denis B, Bougnoux ME, Lanternier F, Lecuit M, Lortholary O. Serum aspergillus galactomannan for the management of disseminated histoplasmosis in AIDS. Am J Trop Med Hyg. 2012;87(2):303-5.
31. Theel ES, Harring JA, Dababneh AS, Rollins LO, Bestrom JE, Jespersen DJ Reevaluation of commercial reagents for detection of Histoplasma capsulatum antigen in urine. J Clin Microbiol. 2015;53(4):1198-203.

32. Freifeld A, Proia L, Andes D, Baddour LM, Blair J, Spellberg B, et al. Voriconazole use for endemic fungal infections. Antimicrob Agents Chemother. 2009;53(4):1648-51.

33. Morlat P. Prise en charge médicale des personnes vivant avec le VIH. Recommandation du groupe d'experts 2013; La documentation française:p268.

\section{Ready to submit your research? Choose BMC and benefit from:}

- fast, convenient online submission

- thorough peer review by experienced researchers in your field

- rapid publication on acceptance

- support for research data, including large and complex data types

- gold Open Access which fosters wider collaboration and increased citations

- maximum visibility for your research: over $100 \mathrm{M}$ website views per year

At BMC, research is always in progress.

Learn more biomedcentral.com/submissions 